\title{
Deep learning to mobile hypermedia and multimedia
}

\author{
Xiaoxian Yang ${ }^{1} \cdot$ Li Kuang $^{2}$
}

Accepted: 19 January 2022

(C) The Author(s), under exclusive licence to Springer Science+Business Media, LLC, part of Springer Nature 2022

For mobile hypermedia and multimedia, arm-based smartphones, laptops, wearables, and other smart devices are everywhere, which have tens of billions or even hundreds of billions of data and information. The deep learning method has made significant breakthroughs in many areas of artificial intelligence. We receive many submissions and finally select six papers for this special issue. A summary of these papers is outlined below.

In the paper entitled "Boosting and Rectifying Few-shot Learning Prototype Network for Skin Lesion Classification Based on the Internet of Medical Things" by Junsheng Xiao et. al, A novel few-shot prototype network was proposed to address the shortage of annotated samples based on IoMT in this study. The experiment on mini-ImageNet and mini-ISIC demonstrates the effectiveness of the proposed method. The author also performed an ablation experiment of different modules of our method, which demonstrates that each component is beneficial for the entire network.

In the paper entitled "TkTC: A Framework for Top-k Text Classification of Multimedia Computing in Wireless Networks " by Kai Wang et al., due to the complex semantic information of the text, traditional text classification that aims to predict the most possible label among all labels may not predict the correct label for the text, and they present TkTC: a framework for top-k text classification, where a novel loss function considers the position of ground truth label and the number of prediction simultaneously. Our method could effectively improve the

Xiaoxian Yang

xxyang@sspu.edu.cn

Li Kuang

kuangli@csu.edu.cn

1 School of Computer and Information Engineering, Shanghai Polytechnic University, Shanghai, China

2 School of Computer Science and Engineering, Central South University, Changsha, China accuracy of top-k text classification and make the correct label have a higher rank.

In the paper entitled "Privacy-Preserving Federated Learning Framework in Multimedia Courses Recommendation" by Yangjie Qin, M.D et al. the authors provide a framework for federated recommendation model learning to address the aforementioned concerns. The authors employ an attention-based hierarchical reinforcement learning model to determine which parts of user data are crucial to targeting in the proposed method. To protect privacy, they aggregate models and coordinate round demarcation using a federated learning system. The experimental results show that the FedMCsRes can perform well in real data set. Compared with another nonfederated method, it can seem to use accuracy to replace secure.

In the paper entitled "A Novel Edge Computing Architecture For Intelligent Coal Mining System” by Zhe Bing et. al, the author propose a novel edge computingbased intelligent processing architecture that integrates Internet of Things (IoT), fifth-generation (5G), and Edge computing technologies for the coal mining intelligent system. Experiments are conducted on a deep learningbased video fire prediction algorithm The results show the effectiveness of the novel method using real colliery data.

In the paper entitled "CCP-Federated Deep Learning Based on User Trust Chain in Social IoV" by PengCheng Zhao et. al, Federated learning is widely used in the context of wireless networks to protect sensitive user data. To cope with the issues of low communication efficiency and high computational cost. The author proposes the Conditional Choice Probability (CCP)-Federated Deep Learning (FDL) algorithm based on the user trust chain. This algorithm introduces inter-user trust elements to characterize the vehicle connection network from a vehicle-user relationship perspective. It computes the node conditional choice trust probability based on the single-way trust atomic chain and circular chain of user nodes. Local model interactions are then performed to complete the decentralized federated deep learning framework. The experiments proved the 
strict convergence of the algorithm and verified the effectiveness of the proposed CCP-FDL algorithm.

In the paper entitled "Web APIs Recommendation with Neural Content Embedding for Mobile Multimedia Computing" by Yueshen Xu et al. a new prediction model (MFAS) is proposed, which uses the hidden features between users and APIs to predict whether users follow the API or not by matrix factorization. On this basis, the similarity between APIs is also calculated by word $2 \mathrm{vec}$, and the influence of the similarity between APIs on the prediction results is also added into the improved formula. The experimental results show that our method achieves high performance.

The Guest Editors would like to express their deep gratitude to all the authors who have submitted their valuable contributions, and to the numerous and highly qualified anonymous reviewers. We also thank the Edit-inChief, Dr. Imrich Chlamtac, for his supportive guidance during the entire process. We also would like to thank the support from the National Natural Science Foundation of China (No. 61902236). We think that the selected contributions, which represent the current state of the art in the field, will be of great interest to the community.

Publisher's Note Springer Nature remains neutral with regard to jurisdictional claims in published maps and institutional affiliations.

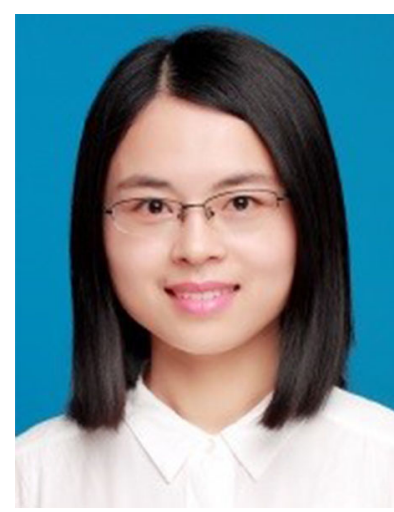

Xiaoxian Yang is currently an associate professor at Shanghai Polytechnic University, China. Her research interests include business process management, formal verification, wireless networks, and mobile healthcare. She has published papers in academic journals, such as IEEE TITS, IEEE TCSS, ACM TOMM, ACM TOIT, DCN, FGCS, MONET, IJSEKE, FGCS, and COMNET. She has also worked as a guest editor for MONET, WINE, and JIT and served as a reviewer for IEEE TII, IEEE T-ITS, IEEE T-ASE, IEEE JBHI, FGCS, PPNA, etc.

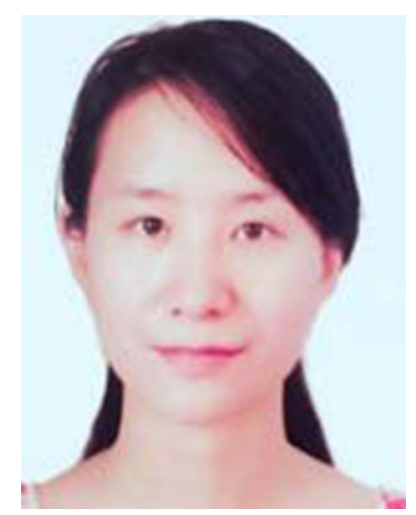

Li Kuang received a Ph.D. in Computer Science from Zhejiang University, China, in 2009. She is currently a professor at the School of Computer Science and Engineering, Central South University. Her research interests include service computing, mobile computing, and privacy preservation. 